\title{
EXISTENCE OF SOLUTIONS \\ IN THE SENSE OF DISTRIBUTIONS \\ OF ANISOTROPIC NONLINEAR ELLIPTIC EQUATIONS WITH VARIABLE EXPONENT
}

\author{
Mohamed Badr Benboubker - Houssam Chrayteh \\ Hassane HJiaj - Chinab YaZOUgh
}

\begin{abstract}
The aim of this paper is to study the existence of solutions in the sense of distributions for a strongly nonlinear elliptic problem where the second term of the equation $f$ is in $W^{-1, \vec{p}^{\prime}(\cdot)}(\Omega)$ which is the dual space of the anisotropic Sobolev $W_{0}^{1, \vec{p}(\cdot)}(\Omega)$ and later $f$ will be in $L^{1}(\Omega)$.
\end{abstract}

\section{Introduction}

Let $\Omega$ be a bounded domain of $\mathbb{R}^{N}(N \geq 2)$ with smooth boundary $\partial \Omega$. For the variable vectorial exponent $\vec{p}(\cdot)=\left(p_{0}(\cdot), \ldots, p_{N}(\cdot)\right)$, we assume that for $i=0, \ldots, N$, the functions $p_{i}(x) \in \mathcal{C}_{+}(\bar{\Omega})$ (defined in Section 2), where

$$
p_{0}(x) \geq \max \left\{p_{i}(x), i=1, \ldots, N\right\}, \quad \text { for any } x \in \bar{\Omega} \text {. }
$$

2010 Mathematics Subject Classification. Primary: 35J66, 46E35; Secondary: 35D30.

Key words and phrases. Anisotropic Sobolev spaces, variable exponent, strongly nonlinear elliptic equations, boundary value problems, solution in the sense of distributions. 
Our aim is to prove the existence of solutions in the sense of distributions to the anisotropic nonlinear elliptic problem:

$$
\begin{cases}-\sum_{i=1}^{N} \partial_{x_{i}} a_{i}(x, u, \nabla u)+g(x, u, \nabla u)+d(x)|u|^{p_{0}(x)-2} u=f & \text { in } \Omega \\ u=0 & \text { on } \partial \Omega\end{cases}
$$

where the right-hand side $f$ is in $W^{-1, \vec{p}^{\prime}(\cdot)}(\Omega)$ which is the dual space of the anisotropic Sobolev space $W_{0}^{1, \vec{p}(\cdot)}(\Omega)$ and later $f$ will be in $L^{1}(\Omega)$. The positive function $d(x)$ belong to $L^{\infty}(\Omega)$, and there exists a constant $d_{0}>0$ such that $d(x) \geq d_{0}$ almost everywhere in $\Omega$.

We assume that for $i=1, \ldots, N$ the function $a_{i}: \Omega \times \mathbb{R} \times \mathbb{R}^{N} \mapsto \mathbb{R}$ is Carathéodory function (i.e. measurable with respect to $x$ in $\Omega$ for every $(s, \xi)$ in $\mathbb{R} \times \mathbb{R}^{N}$ and continuous with respect to $(s, \xi)$ in $\mathbb{R} \times \mathbb{R}^{N}$ for almost every $x$ in $\Omega$ ) which satisfies the following conditions:

$$
\begin{array}{ll}
\left|a_{i}(x, s, \xi)\right| \leq \beta\left(K_{i}(x)+|s|^{p_{i}(x)-1}+\left|\xi_{i}\right|^{p_{i}(x)-1}\right) & \text { for } i=1, \ldots, N, \\
a_{i}(x, s, \xi) \xi_{i} \geq \alpha\left|\xi_{i}\right|^{p_{i}(x)} & \text { for } i=1, \ldots, N,
\end{array}
$$

$a_{i}(\cdot, \cdot, \cdot)$ is strictly monotone, i.e. for all $\xi=\left(\xi_{1}, \ldots, \xi_{N}\right)$ and $\xi^{\prime}=\left(\xi_{1}^{\prime}, \ldots, \xi_{N}^{\prime}\right)$ in $\mathbb{R}^{N}$, we have

$$
\left(a_{i}(x, s, \xi)-a_{i}\left(x, s, \xi^{\prime}\right)\right)\left(\xi_{i}-\xi_{i}^{\prime}\right)>0, \quad \text { for } \xi_{i} \neq \xi_{i}^{\prime},
$$

for almost every $x \in \Omega$ and all $(s, \xi) \in \mathbb{R} \times \mathbb{R}^{N}$, where $K_{i}(\cdot)$ is a non-negative function lying in $L^{p_{i}^{\prime}(\cdot)}(\Omega)$ where $1 / p_{i}(x)+1 / p_{i}^{\prime}(x)=1$ and $\alpha, \beta>0$ are two positive constants.

Note that, Gwiazda et al. in [17] studied a steady and in [18] a dynamic model for non-Newtonian fluids under an additional strict monotonicity assumption on the operator. The authors used Young measure techniques in place of a monotonicity method. Moreover, a version of the Minty-Browder trick adapted to the setting of generalized Orlicz spaces was introduced in [27] (see also [19]) in framework of non-Newtonian fluids.

The nonlinear term $g(x, s, \xi)$ is a Carathéodory function which satisfies

$$
\begin{aligned}
& g(x, s, \xi) s \geq 0 \\
& |g(x, s, \xi)| \leq b(|s|)\left(c(x)+\sum_{i=1}^{N}\left|\xi_{i}\right|^{p_{i}(x)}\right),
\end{aligned}
$$

where $b(\cdot): \mathbb{R}^{+} \rightarrow \mathbb{R}^{+}$is a continuous non-decreasing function, and $c(\cdot): \Omega \rightarrow$ $\mathbb{R}^{+}$with $c(\cdot) \in L^{1}(\Omega)$.

In view of (1.7), the Carathéodory function $g(x, u, \nabla u)$ does not define a mapping from $W_{0}^{1, \vec{p}(\cdot)}(\Omega)$ into its dual, but from $W_{0}^{1, \vec{p}(\cdot)}(\Omega) \cap L^{\infty}(\Omega)$ into $L^{1}(\Omega)$ (see also [9]). 